\title{
Efecto del ultrasonido en la digestibilidad in vitro de las proteínas contenidas en la harina de trigo
}

\section{Gelvez Ordoñez Víctor M ${ }^{1}$, Luna Nidia J ${ }^{2}$, Campo Vera Yesenia ${ }^{3}$.}

${ }^{1}$ Universidad de Pamplona.Programa Ingeniería de Alimentos. Facultad de Ingenierías y Arquitectura. Sede Villa de Rosario.

Universidad de Pamplona. Programa Nutrición y Dietética. Facultad de Salud. Pamplona. Colombia.

3Universidad Francisco de Paula Santander. Programa Ingeniería Agroindustrial. Grupo de Investigación en Ciencia y Tecnología Agroindustrial (GICITECA). Cúcuta. Colombia.

\section{Resumen}

En las últimas décadas la industria busca la transformación e innovación de los alimentos, sin modificar sus propiedades nutricionales, asegurando su calidad e inocuidad. El objetivo de este trabajo fue evaluar el efecto del Ultrasonido sobre la digestibilidad in vitro de las proteínas contenidas en la harina de trigo, para lo cual se suspendió la harina en agua y se sometió a termosonicación $(40 \mathrm{KHz})$ a diferentes temperaturas $\left(40,50\right.$ y $\left.60^{\circ} \mathrm{C}\right)$ durante 15 y 30 minutos. La digestibilidad in vitro de las proteínas se determinó utilizando un sistema trienzimático (tripsina, quimotripsina y peptidasa). Se encontró un incremento significativo $(p<0,05)$ en la digestibilidad de las muestras tratadas a $40 \mathrm{KHz}$ $150^{\circ} \mathrm{C} / 15$ min alcanzando un valor de $85,92 \pm 0,11 \%$ con respecto a los demás muestras. No se observó ninguna variación en los demás tratamientos con respecto a la muestra control. Se registró un promedio del valor de la digestibilidad, en las muestras tratadas y las control, que oscila entre 84,50 a $86,00 \%$. Los resultados obtenidos podrían deberse a la constitución básica de la harina de trigo; ya que la molienda de los granos aumenta la superficie para el ataque enzimático y el fenómeno de cavitación causado por el US que aumenta la temperatura de las proteínas desnaturalizándolas y mejorando la digestibilidad. Se puede concluir que el efecto del US mejora la digestibilidad proteica de la harina lo que permiten aumentar su valor nutricional y su calidad de los alimentos.

Palabras clave: Ultrasonido, digestibilidad, trigo, proteína. 


\section{Effect of ultrasound in vitro digestibility of protein contained in wheat flour}

\section{Abstract}

In recent decades, the industry seeks the transformation and innovation of food without altering its nutritional properties, ensuring their quality and harmlessness. The aim of this study was to evaluate the effect of the US on the in vitro digestibility of the proteins contained in wheat flour, for which the flour was suspended in water and subjected to thermosonication $(40 \mathrm{KHz})$ at temperatures of 40,50 and $60{ }^{\circ} \mathrm{C}$ and each of them for 15 and 30 minutes. The in vitro protein digestibility was determined using a tree enzymatic system (trypsin, chymotrypsin and peptidase). A significant increase $(p<0.05)$ in the US for treatment $40 \mathrm{KHz}$ to $50{ }^{\circ} \mathrm{C}$ for 15 minutes was found reaching a value of $85.92 \pm 0.11 \%$ with respect to the other samples. No variation was observed in the other treatments compared to the control sample, averaging ranging from 84.50 to $86.00 \%$ of the value of the in vitro digestibility of the treated samples and not with US. The results could be due to the basic constitution of wheat flour; as grinding grains increases the surface for enzymatic attack and the cavitation phenomenon caused by the US increases the temperature of the denaturing proteins and improving the digestibility. It concludes that the effect of the improvement in US vitro protein flour, features to increase the nutritional value and quality of food digestibility.

Keywords:Ultrasound, digestibility, wheat, protein.

\footnotetext{
*Para citar este articulo: Gelvez Ordoñez V M, Luna NJ, Campo Vera Y.Efecto del ultrasonido en la digestibilidad in vitro de las proteínas contenidas en la harina de trigo. Revista. Bistua.2015.13(1):81-91
}

+ Autor para el envió de correspondencia y la solicitud de las separatas: Víctor M. Gelvez Ordoñez.Universidad de Pamplona,.Programa Ingeniería de Alimentos. Facultad de Ingenierías y Arquitectura. Sede Villa de Rosario. A.A. 50576, Autopista Internacional Vía Los Álamos Villa Antigua.Tels: (57+7) 5706966 (Fax) - 5703742. Cúcuta, Colombia. Número telefónico: 312.4829690.email:vmgelvez@gmail.com 


\section{Introducción}

El trigo es uno de los tres cereales más producidos globalmente, junto al maíz y el arroz y el más ampliamente consumido por el hombre en la civilización occidental desde la antigüedad(1). El grano del trigo es utilizado para hacer harina, harina integral, sémola, cerveza y una gran variedad de productos. ${ }^{(2)}$

El incremento de la producción hace que el trigo sea el primer cereal desde el punto de vista comercial, muy importante en el mercado, anualmente se producen $100 \mathrm{~kg}$ de trigo por cada habitante en el mundo y casi toda su producción se destina a la alimentación humana. ${ }^{(3)}$

El valor nutricional de un alimento se basa en su composición química y en la capacidad del organismo que lo consume para digerir y absorber los nutrientes y la energía en él contenida. ${ }^{(4)(5)}$

La combinación de conocimientos sobre composición química y digestibilidad de un ingrediente, a utilizar en la formulación de una ración, permite precisar los cálculos sobre su contribución en términos de nutrientes y de energía y de igual manera, estimar la proporción no digerible que será eliminada con las heces; la formulación de dietas con base en estos dos criterios es la clave para lograr raciones nutricionalmente eficientes y de reducido impacto ambiental. (5)(6)(7)(8)(9)

Las proteínas ocupan un lugar primordial entre los principios alimentarios, los datos analíticos sobre la composición aminoacídica y su digestibilidad permiten determinar su valor nutricional. (10)(11)

Factores como el calor, pueden incrementar la digestibilidad al modificar la estructura terciaria y secundaria o sea al desnaturalizar la proteína. La solubilidad de las proteínas aumenta con la temperatura entre 0 y $40-50^{\circ} \mathrm{C}$; por el contrario los tratamientos térmicos mal aplicados (altas temperaturas durante tiempos prolongados) provocan la formación de enlaces indigestibles entre aminoácidos ó entre aminoácidos y azúcares (reacción de Maillard), lo que reduce su digestibilidad. ${ }^{(12)}$

El Ultrasonido (US) se define como la energía generada en ondas sónicas de $20 \mathrm{KHz}$ o más vibraciones por segundo. ${ }^{(13)}$ Con los intervalos de 20 a $100 \mathrm{kHz}$ (baja frecuencia) debido a la cavitación producida durante el tratamiento de los alimentos se alcanzan cambios químicos y físicos deseables en los productos. ${ }^{(14)}$ 
El tratamiento con US $(20-40 \mathrm{KHz})$ aumenta la solubilidad de las proteínas por cambios estructurales en su conformación, los residuos de aminoácido hidrófilos son orientados hacia el agua. ${ }^{(15)}$

En la evalución del efecto del US en la glicolisis y oxidación de proteínas de suero, se observó que la cavitación causa aceleración en la reacciones de Maillard formando estructuras de tipo amilosas y mejora la estabilidad de la estructura de las proteínas; lo que permite que el tiempo de procesamiento sea corto y condiciones menos agresivas, preservando la estructura de las proteínas y reduciendo al mínimo la agregación de estás ${ }^{(16) .}$

El objetivo de este estudio fue evaluar el efecto de la termosonicación (40 $\mathrm{KHz})$ a diferentes temperaturas (40, 50 y $60^{\circ} \mathrm{C}$ ) durante 15 y $30 \mathrm{~min}$ sobre la digestibilidad in vitro de las proteínas contenidas en la harina de trigo.

\section{MATERIALES Y MÉTODOS Materia prima.}

Se utilizó harina de Trigo adquirida en el mercado principal de la ciudad de Pamplona (Norte de Santander.)

Las enzimas utilizadas fueron: Tripsina pancreática (tipo IX,sigma T0134 actividad $17.000 \mathrm{u} / \mathrm{mg}$ de 84

Bistua Revista de la Facultad de Ciencias Básicas.Universidad de Pamplona.ISSN 01204211 proteína. Quimiotripsina bovina pancreática ( Typo II, sigma C 4129 actividad $56 \mathrm{u} / \mathrm{mg}$ de poder y Peptidasa (grade K, Sigma P-7500 actividad $100 \mathrm{u} / \mathrm{g}$ de poder).

Los test de las muestras de la solución trienzimática (tripsina, Quimiotripsina y Peptidasa) fueron preparados con un estándar de caseinato de sodio (sigma 8654).

\section{Preparación de las muestras}

La reconstitución de la harina (trigo) se realizó mezclándola con agua (50 $\%$ en base al peso seco) a $25^{\circ} \mathrm{C}$, se agitó constantemente hasta obtener una mezcla homogénea evitando la formación de grumos. ${ }^{(17)}$ Posteriormente se envasó $50 \mathrm{~mL}$ de cada una de las muestras en bolsas de polietileno de baja densidad y se empacaron al vacío (90\%).

\section{Tratamiento con ultrasonido}

Se empleó un equipo Branson 1510 $(40 \mathrm{KHz})$ y como medio de transmisión agua desionizada, el tratamiento de US se llevó a cabo a temperatura de 40,50 y $60^{\circ} \mathrm{C}$, durante 15 y 30 minutos, dando lugar a seis tratamientos diferentes. La muestra control fue una mezcla homogénea de cada harina de trigo con agua.

\section{Determinación de Digestibilidad in vitro.}


85

Determinación de contenido de Nitrogeno total.

Los contenidos de nitrógeno total de las muestras de harina fueron determinados en el laboratorio de la Maestría en Ciencia y Tecnología de los Alimentos de la Universidad de Pamplona siguiendo lo establecido en el método Kjeldahl. ${ }^{(18)}$

Preparación de las muestras para digestibilidad in vitro.

Para determinar la digestibilidad in vitro se empleó un liofilizador pederssen eggum $\mathrm{pH}$ stat procedure; Model 100 SRC-8 en bancos unitarios (virtis co.,Gardner,NY 12525). Las muestras fueron puestos en bandejas de $30^{*} 61 \mathrm{cms}$ de (2 a $3 \mathrm{~L}$ por bandeja hasta 24 bandejas) y congelados a $-50^{\circ} \mathrm{C}$ de Temperatura bajo vacío de 5-10 $\mu \mathrm{m}$ y temperatura final de $25^{\circ} \mathrm{C}$. Las muestras fueron secadas en aire por 12 horas y tamizadas en una malla de $20 \mathrm{~cm}$ de grosor.

Preparación de soluciones enzimáticas para digestibilidad in vitro.

Se prepararon $90 \mathrm{ml}$ de solución trienenzimática que contenía 23.100 unidades de tripsina, 186 unidades de quimiotripsina, y 0.052 unidades de peptidasa. Se ajustó el $\mathrm{pH}$ hasta 8.0 manteniendo una temperatura de $37^{\circ} \mathrm{C}$ por dos minutos. Posteriormente la solución fue almacenada a $0^{\circ} \mathrm{C}$. La actividad Bistua Revista de la Facultad de Ciencias Básicas. Universidad de Pamplona.ISSN 01204211 enzimática fue determinada utilizando $10 \mathrm{~mL}$ de la suspensión de caseinato de sodio (1 $\mathrm{mg} \mathrm{N} / \mathrm{mL}$ de agua) los cuales fueron introducidos en un vaso que se mantuvo a $37^{\circ} \mathrm{C}$ y se le ajusto el $\mathrm{pH}$ hasta 8.0 antes de agregar 1 $\mathrm{mL}$ de solución trienzimática. La actividad enzimática se determinó por la cantidad de $0.1 \mathrm{~N} \mathrm{NaOH}$ requerido para mantener el $\mathrm{pH}$ a 7.98 por 10 minutos. El porcentaje digestibilidad (TD) fue calculada teniendo en cuenta la siguiente fórmula:

$$
\mathrm{TD}=76.14+47.77 B
$$

Donde $B=\mathrm{ml} 0.1 \mathrm{~N} \mathrm{NaOH}$ agregado. La digestibilidad del caseinato de sódio se determinó como 98\%.

\section{Digestibilidad de los test de proteína.}

La digestibilidad de las muestras de proteína contenida en las soluciones de harina se hizo por triplicado y el caseinato de sodio como control fueron determinados de la siguiente forma: La muestra que contiene de 10 $\mathrm{mg}$ de nitrógeno fue disuelta en 2.5 $\mathrm{mL}$ de agua; posteriormente $2.5 \mathrm{~mL}$ de $0.2 \mathrm{~N} \mathrm{NaOH}$ fueron adicionados a la solución y se almacenó a $37^{\circ} \mathrm{C}$. Después de 30 minutos, $5.0 \mathrm{~mL}$ de $\mathrm{HCL}(0.075 \mathrm{~N})$ fue añadido y se ajusto el $\mathrm{pH}$ fue a 8.0. Luego se adicionó $1 \mathrm{~mL}$ de la solución trienzimática. Se calculó la cantidad de $0.1 \mathrm{~N} \mathrm{NaOH}$ requerido para 
mantener el pH hasta 7.98 durante 5 minutos.

\section{Análisis estadístico}

Se analizaron las diferencias significativas entre los resultados obtenidos con cada tratamiento, mediante prueba de análisis de varianza (ANOVA) y prueba post hoc de Diferencias Mínimas Significativas (DMS) del $5 \% \quad(p<0,05)$ usando el programa SPSS versión 19.0.Todos los experimentos se hicieron por triplicado.

\section{RESULTADOS}

En la figura 1, se presentan los resultados del efecto del US sobre la digestión in vitro de la proteína contenida en la harina de trigo, donde se evidencia incremento significativo $(p<0,05)$ de una unidad en las muestras sometidas a tratamiento de $40 \mathrm{KHz} / 50^{\circ} \mathrm{C} / 15$ minutos. No se observa ninguna variación significativa $(p<0,05)$ en los demás tratamientos con respecto a la muestra control. Se Obtuvo un promedio de la digestibilidad in vitro de la proteína contenida en todas las muestras que oscila de 84,50 a $86,00 \%$.

Los análisis estadísticos indican que se presentaron diferencias significativas al $95 \%$ entre todos los tratamientos y con la prueba de DMS se pueden asociar en tres grupos homogéneos (figura 1). 86
Las muestras tratadas a $40 \mathrm{KHz}$ $150^{\circ} \mathrm{C} / 15$ minutos presentan un $86 \%$ de digestibilidad in vitro lo cual representa un aumento del $6 \%$ de ésta con respecto a la muestra control.

\section{DISCUSIÓN}

Los resultados de la digestibilidad in vitro obtenidos en el presente estudio $(86 \%)$ son mayores a los obtenidos en harina de trigo sin tratamiento $(42,56 \pm 11,45 \%)$, este último resultado es atribuido al contenido de amilopectina $(80 \%)$ presente en el trigo en su forma nativa, en comparación con otras fuentes vegetales. ${ }^{(19)(20)(21)}$

El aumento de la digestibilidad (6\%) es debido al fenómeno de cavitación propio del tratamiento con US; que al aumentar la temperatura y la presión interna en el alimento genera la salida de enzimas y péptidos que facilitan la digestión proteica. (22)(23)(24) EI US favorece desdoblamiento de las proteínas y la hidrólisis de éstas. ${ }^{(25)(15)}$

En los resultados obtenidos del porcentaje de digestibilidad in vitro de las disoluciones estudiadas se puede observar que en las muestras tratadas con $40 \mathrm{KHz} / 50^{\circ} \mathrm{C} / \min$ y 40 $\mathrm{KHz} / 60^{\circ} \mathrm{C} / 15$ min el efecto del tratamiento es mayor pues los valores de la digestibilidad aumentan, 
lo anterior debido a que la molienda de los granos aumenta la superficie de contacto y la humectación en este caso. A su vez el fenómeno de cavitación producido durante el tratamiento desnaturaliza las proteínas mejorando su digestibilidad. (25)

La calidad nutricional de una proteína que más allá de una composición balanceada en aminoácidos, depende de su facilidad para hidrolizarse y que los aminoácidos liberados sean asimilados e ingresen al torrente sanguíneo.(26) Se observó en este estudio que al desnaturalizarse las proteínas por efecto del tratamiento con US se eleva la calidad nutricional ya que las proteínas de la harina de trigo aumenta su digestibilidad por lo tanto su calidad nutricional.

Al comparar los resultados encontrados en este caso con otros estudios sobre productos de la misma textura como la harina de arroz (Digestibilidad 54,12 $\pm 14,90 \%$ ) se observa que el tratamiento con ultrasonido eleva la calidad nutricional de la harina de trigo. ${ }^{(19)}$

Otros estudios han demostrado el efecto del US $(37 \mathrm{KHz} / 20)$ y $45^{\circ} \mathrm{C} / 5 \mathrm{a}$ $15 \mathrm{~min}$ ) sobre las proteínas del huevo entero líquido, demostrando que cuando la muestra es tratada a temperaturas elevadas por cortos tiempos el incremento de la 87

Bistua Revista de la Facultad de Ciencias Básicas.Universidad de Pamplona.ISSN 01204211 digestibilidad es directamente proporcional a la temperatura; mientras que a baja temperatura el incremento en dicha digestibilidad es proporcional al aumento del tiempo(27). Lo anterior demuestra el aumento de la digestibilidad de la harina de trigo en los tratamiento con $40 \mathrm{kHz} / 50^{\circ} \mathrm{C} / 15 \mathrm{~min}$.

\section{CONCLUSIONES}

El aumento $(6 \%)$ significativo $(p<0,05)$ en el porcentaje de digestibilidad in vitro de las muestras de harina de trigo tratadas con US $(40 \mathrm{kHz} / 50$ y 60 ${ }^{\circ} \mathrm{C} / 15$ minutos) es debido al fenómeno de cavitación generado durante el tratamiento. Este tratamiento permite aumentar el valor nutricional y la calidad de las muestras estudiadas.

\section{Referencias bibliogràficas}

1. Bednar GE, Patil AR, Murray SM, Grieshop CM, Merchen NR, Fahey GC. Starch and fiber fractions in selected food and feed ingredients affect their small intestinal digestibility and fermentability and their large bowel fermentability in vitro in a canine model. J Nutr [Internet]. 2001 Feb 1 [cited 2015 Sep 21];131(2):276-86. Available from:

http://jn.nutrition.org/content/13 1/2/276.abstract 
2. Forero DG. Almacenamiento de Granos. UNAD, Fac Ciencias Agrar Bogotá. 2000;25.

3. Dendy DA V, Dobraszczyk BJ. Cereals and cereal products: chemistry and technology. Aspen publishers; 2001.

4. NRC (National Research Council). Health Effects of Ingested Fluoride. 1993. Washington, DC: National Academy Press.

5. Vásquez-Torres W, Yossa Ml, Gutiérrez-Espinosa MC. Digestibilidad aparente de ingredientes de origen vegetal y animal en la cachama. Pesq.agropec.bras., Brasília 2013;48(8):920-927.

6. Sullivan JA, Reigh RC. Apparent digestibility of selected feedstuffs in diets for hybrid striped bass (Morone saxatilis o $\mathrm{X}$ Morone chrysops §). Aquaculture [Internet]. 1995 Dec [cited 2015 Sep 21];138(14):313-22. Available from: http://www.sciencedirect.com/sc ience/article/pii/0044848695010 $\underline{718}$

7. Laining A, Ahmad T, Williams K. Apparent digestibility of selected feed ingredients for humpback grouper, Cromileptes altivelis. Aquaculture [Internet]. 2003 Mar [cited 2015 Sep 21];218(1-4):529-38. Available from:
88

http://www.sciencedirect.com/science article/pii/S0044848602004635

8. Köprücü K, Özdemir Y. Apparent digestibility of selected feed ingredients for Nile tilapia (Oreochromis niloticus). Aquaculture [Internet]. 2005 Nov [cited 2015 Jul 14];250(1-2):308-16. Available from: http://www.sciencedirect.com/sc ience/article/pii/S004484860400 7148

9. Guillaume J, Blanco AS. Nutrición y alimentación de peces y crustáceos [Internet]. Mundi-Prensa; 2004. Available from:

https://books.google.com.co/bo oks? id=k3A44mLcKnAC.

10. Otero MA. Proteína unicelular para consumo humano. La Habana Editor CientíficoTécnica. 1985;87-97.

11. Morris Quevedo HJ, Carrillo Farnés O, Bermúdez Savón RC. Enfoque integral en la utilización de los métodos químicos de evaluación de la calidad proteica. Rev Cuba Salud Pública. 1999, Editorial Ciencias Médicas; 2003;29(1):42-7.

12. Cheftel JC, Cuq JL, Lorient D. Aminoácidos, péptidos y proteínas. Química los Aliment 
Acribia Zaragoza, España. 1993;275414.

13. Hoover DG. Ultrasound. J Food Sci. Wiley Online Library; 2000;65(s8):93-5.

14. McClements DJ. Advances in the application of ultrasound in food analysis and processing. Trends Food Sci Technol. Elsevier; 1995;6(9):293-9.

15. Moulton KJ, Wang LC. A PilotPlant Study of Continuous Ultrasonic Extraction of Soybean Protein. J Food Sci. Wiley Online Library; 1982;47(4):1127-9.

16. Perusko $M$, Al-Hanish $A$, Velickovic TC, Stanic-Vucinic D. Macromolecular crowding conditions enhance glycation and oxidation of whey proteins in ultrasound-induced Maillard reaction. Food Chem. Elsevier; 2015;177:248-57.

17. Jambrak AR, Lelas $\mathrm{V}$, Mason TJ, Krešić G, Badanjak M. Physical properties of ultrasound treated soy proteins. J Food Eng [Internet]. 2009 Aug [cited 2015 Sep 21];93(4):38693. Available from: http://www.sciencedirect.com/sc ience/article/pii/S026087740900 065X.

18. International A. Official methods of analysis of AOAC
89

International.

AOAC International; 2005.

19. Perera E, Rodríguez-Viera L, Casuso A. Digestibilidad in vitro de carbohidratos en la langosta espinosa Panulirus argus (Latreille, 1804). 2013.

20. Cruz-Suarez LE, Ricque-Marie D, Pinal-Mansilla JD, WescheEbelling P. Effect of different carbohydrate sources on the growth of Penaeus vannamei: economical

Aquaculture. impact. 1994;123(3):349-60.

21. Cuzon G, Rosas C, Gaxiola G, Taboada G, Van Wormhoudt A. Utilization of carbohydrates by shrimp1. 2000.

22. Postema $M$, Van Wamel $A$, Lancée $\mathrm{CT}$, De Jong $\mathrm{N}$. Ultrasound-induced encapsulated microbubble phenomena. Ultrasound Med Biol. Elsevier; 2004;30(6):82740.

23. Wibetoe G, Takuwa DT, Lund W, Sawula G. Coulter particle analysis used for studying the effect of sample treatment in slurry sampling electrothermal atomic absorption spectrometry. Fresenius $J$ Anal Chem [Internet]. Springer-Verlag; 1999;363(1):46-54. Available from: 
http://dx.doi.org/10.1007/s0021

90

$\underline{60051136}$

24. López-Ferrer D, Capelo JL, Vázquez J. Ultra Fast Trypsin Digestion of Proteins by High Intensity Focused Ultrasound. J Proteome Res [Internet]. American Chemical Society; 2005 Oct 1;4(5):1569-74. Available from: http://dx.doi.org/10.1021/pr0501 $12 \mathrm{v}$

25. Pinciroli M. Proteínas de arroz: propiedades estructurales y funcionales. Facultad de Ciencias Agrarias y Forestales; 2011.

26 Gelvez VM, Hernandez E. Efecto del ultrasonido y campo magnéticos sobre las propiedades físicas, químicas, reológicas y microbiológicas del huevo entero liquido. Universidad de Pamplona; 2010. 
91

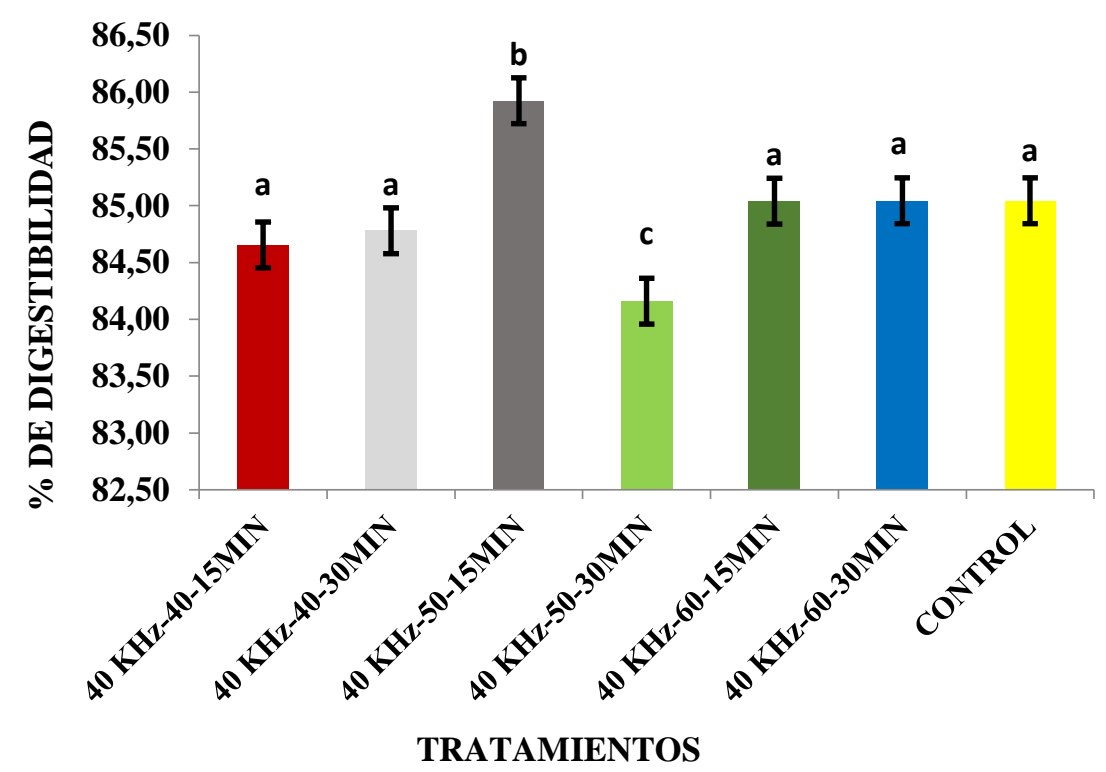

*Letras Iguales entre columnas no hay diferencias mínimas significativas (DMS-p<0.05) entre tratamientos.

Figura 1. Porcentaje de digestibilidad en las muestra de harina de trigo sometidas a termosonicación.

Gelvez Ordoñez Víctor M .Ph.D. Profesor Asociado. Universidad de Pamplona.Programa Ingeniería de Alimentos. Facultad de Ingenierías y Arquitectura. Sede Villa de Rosario.

Luna Nidia J -Ms.C. Profesora Asistente.Universidad de Pamplona. Programa Nutrición y Dietética. Facultad de Salud. Pamplona. Colombia.

Campo Vera Yesenia.Ms.C. Universidad Francisco de Paula Santander. Programa Ingeniería Agroindustrial. Grupo de Investigación en Ciencia y Tecnología Agroindustrial (GICITECA). Cúcuta. Colombia. 
Bistua:Revista de la Facultad de Ciencias Básicas.2015.13(1):81-91

Bistua Revista de la Facultad de Ciencias Básicas.Universidad de Pamplona.ISSN 01204211 